\title{
GENERATION OF AN ACOUSTIC PULSE BY A BAFFLED CIRCULAR PISTON
}

\author{
by P. CHADWICK and G. E. TUPHOLME
}

(Received 5th September 1966)

\section{Introduction}

The generation of acoustic disturbances in a fluid of semi-infinite extent by the motion of a circular piston surrounded by a plane rigid baffle has been studied quite extensively (see (1), (2), (3), (4) and further references given in these papers). Attention has been devoted mainly to the case in which the piston executes a harmonic oscillation of small amplitude, and only comparatively recently has Oberhettinger (2) demonstrated how the time-harmonic solution can be used to solve the more general problem in which the normal velocity of the piston is an arbitrary function of time. The purpose of the present paper is two-fold. Firstly, we point out that for arbitrary normal motion of the piston the "baffled piston problem" can be solved directly, and in a particularly simple manner, by means of a technique involving integral transforms which has been applied by Mitra (5) and Eason (6) to the study of shear wave propagation in an elastic half-space. Secondly, we give a more detailed account than appears to be available in the literature of the structure of the sound pulse generated by the arbitrary normal motion of a baffled piston.

The problem under consideration may be regarded as a limiting case of the much more complicated situation in which a sound pulse is generated in the fluid outside an impermeable sphere by the radial motion of a cap of the sphere. A detailed investigation of this more difficult problem has been made by one of the present authors (7).

The content of the later sections of this paper is, in brief, as follows. In $\S 2$ the baffled piston problem is formulated and a tentative solution is obtained by the method mentioned above. The verification of the tentative solution is then given in outline. $\S 3$ is concerned with the examination of the structure of the sound pulse and the nature of the wavefronts present in it. The motion of the piston as a whole gives rise to a disturbance (the direct wave) which is confined to a circular cylinder having the piston as its base and headed by a plane wavefront. The discontinuity in the normal velocity of the boundary, associated with the motion of the edge of the piston relative to the baffle, gives rise to a second wave which we term the edge wave. The edge wavefront is a half-torus having as its centre circle the edge of the piston. The inner portion of the edge wave expands into the region of fluid already disturbed by the direct wave and eventually overlaps itself. The direct and edge wavefronts are 
singular surfaces of the flow in the sense that they carry discontinuities in partial derivatives of the velocity potential. Finally, in $\S 4$, we take up the special case in which the piston moves with constant speed. It now becomes possible to determine the pressure field in a simple closed form and graphs are presented showing the variation with time of the pressure at representative points of the fluid and the distribution of pressure on the piston and baffle at various times.

\section{Formulation and solution of the problem}

A semi-infinite expanse of homogeneous compressible fluid is bounded by an infinite impermeable boundary consisting of a circular plane piston of radius $a$ surrounded by a fixed plane baffle. Initially the fluid is everywhere at rest at uniform density $\rho_{0}$ and pressure $p_{0}$ and the piston and baffle are coplanar. Beginning at time $t=0$, an acoustic pulse is generated in the fluid by moving the piston.

Defining cylindrical polar coordinates $(r, \theta, z)$ with the origin $O$ at the centre of the initial position of the piston and with the $z$-axis directed perpendicular to the baffle and into the fluid, we assume that at all times the piston has its centre on the $z$-axis and lies in a plane of constant $z$. It follows that the motion of the piston is specified by its velocity $v(t)$ in the positive $z$-direction and that the acoustic pulse is axisymmetric about the $z$-axis, being described by a velocity potential $\phi$ which is a function of $r, z$ and $t$.

The initial-boundary-value problem for $\phi(r, z, t)$ consists of the wave equation

$$
\frac{\partial^{2} \phi}{\partial r^{2}}+\frac{1}{r} \frac{\partial \phi}{\partial r}+\frac{\partial^{2} \phi}{\partial z^{2}}=\frac{1}{c^{2}} \frac{\partial^{2} \phi}{\partial t^{2}} \text { for } r \geqq 0, z>0, t \geqq 0,
$$

and the subsidiary conditions

$$
\begin{gathered}
\phi=0, \frac{\partial \phi}{\partial t}=0 \text { for } r \geqq 0, z \geqq 0 \text { at } t=0, \\
\frac{\partial \phi}{\partial z}=\left\{\begin{array}{r}
(t) \text { for } r<a, t \geqq 0 \\
0 \text { for } r>a, t \geqq 0
\end{array}\right\} \text { at } z=0,
\end{gathered}
$$

$c$ being the speed of sound in the fluid.

We obtain a tentative solution of the problem (2.1) to (2.3) by applying the one-sided Laplace transform with respect to the time $t$ followed by the Hankel transform of order zero with respect to the radial coordinate $r$ (cf. (8), pp. 125-128). As the tentative solution is verified a posteriori, no justification is given for the various analytic processes employed in its construction.

With the usual notation for Bessel functions of the first kind, let

$$
\begin{aligned}
\tilde{\phi}(z) & =\int_{0}^{\infty} \int_{0}^{\infty} \phi(r, z, t) r J_{0}(\xi r) e^{-s t} d t d r, \\
\bar{v} & =\int_{0}^{\infty} v(t) e^{-s t} d t .
\end{aligned}
$$


Then, multiplying each term in equations (2.1) and (2.3) by $r J_{0}(\xi r) e^{-s t}$, integrating over the quadrant $r \geqq 0, t \geqq 0$, and making use of (2.2), we obtain

$$
\begin{gathered}
\frac{d^{2} \tilde{\phi}}{d z^{2}}-\left(\xi^{2}+\frac{s^{2}}{c^{2}}\right) \delta=0 \text { for } z>0, \\
\frac{d \tilde{\phi}}{d z}=\bar{v} \frac{a}{\xi} J_{1}(\xi a) \text { at } z=0 .
\end{gathered}
$$

The solution of the transformed problem (2.4), (2.5) which remains bounded as $z \rightarrow \infty$ is

$$
\Phi(z)=-\bar{v} \frac{a J_{1}(\xi a)}{\xi\left(\xi^{2}+s^{2} / c^{2}\right)^{\frac{1}{2}}} \exp \left\{-z\left(\xi^{2}+s^{2} / c^{2}\right)^{\frac{1}{t}}\right\}
$$

and formal inversion of the double transform $\phi(z)$ yields

$\phi(r, z, t)=-\frac{a}{2 \pi i} \int_{x-i \infty}^{\kappa+i \infty} \bar{v} e^{s t} d s \int_{0}^{\infty} \frac{J_{1}(\xi a) J_{0}(\xi r)}{\left(\xi^{2}+s^{2} / c^{2}\right)^{\frac{1}{t}}} \exp \left\{-z\left(\xi^{2}+s^{2} / c^{2}\right)^{\frac{1}{1}}\right\} d \xi$,

$\kappa$ being a positive constant with the property that all the singularities of the integrand in the complex s-plane lie to the left of the line $\operatorname{Re} s=\kappa$.

Proceeding now to the simplification of the integral representation (2.6), we first reverse the order of integration and then use a known result ((9), p. 248) for the inverse of the Laplace transform

$$
\left(\xi^{2}+s^{2} / c^{2}\right)^{-\frac{1}{2}} \exp \left\{-z\left(\xi^{2}+s^{2} / c^{2}\right)^{\frac{1}{t}}\right\}
$$

Since $\bar{v}$ is the Laplace transform of $v(t)$, an application of the convolution theorem gives

$$
\begin{aligned}
& \frac{1}{2 \pi i} \int_{x-i \infty}^{x+i \infty} \frac{\bar{v}}{\left(\xi^{2}+s^{2} / c^{2}\right)^{\frac{1}{t}}} \exp \left\{-z\left(\xi^{2}+s^{2} / c^{2}\right)^{\frac{1}{t}}+s t\right\} d s \\
& \quad=c \int_{0}^{t} v(t-\tau) J_{0}\left\{\xi c\left(\tau^{2}-z^{2} / c^{2}\right)^{\frac{1}{2}}\right\} H(\tau-z / c) d \tau \\
& \quad=\int_{z}^{c t} v(t-\eta / c) J_{0}\left\{\xi\left(\eta^{2}-z^{2}\right)^{\frac{1}{2}}\right\} d \eta H(t-z / c),
\end{aligned}
$$

where $H$ is the Heaviside unit function, and the tentative solution (2.6) reduces to $\phi(r, z, t)=-a \int_{z}^{c t} v(t-\eta / c) d \eta \int_{0}^{\infty} J_{1}(\xi a) J_{0}\left\{\xi\left(\eta^{2}-z^{2}\right)^{\frac{1}{t}}\right\} J_{0}(\xi r) d \xi H(t-z / c)$.

This result (with $c=1$ ) can easily be manipulated into the form derived by Oberhettinger ((2), equations (18) and (19)).

The factor $H(t-z / c)$ on the right-hand side of (2.7) implies that $\phi=0$ for $z>c t$. Hence, as would be expected from the general theory of the threedimensional wave equation, the region of disturbed fluid at time $t$ lies between the boundary $z=0$ and the plane $z=c t$.

E.M.S. - $S$ 
The simplification is completed by evaluating the infinite integral in (2.7) with the aid of the result ((10), p. 411)

$$
\begin{aligned}
& \int_{0}^{\infty} J_{1}(\xi a) J_{0}(\xi b) J_{0}(\xi c) d \xi \\
&= \begin{cases}0, & 0 \leqq a \leqq|b-c|, \\
\frac{1}{\pi a} \cos ^{-1}\left\{\left(b^{2}+c^{2}-a^{2}\right) / 2 b c\right\}, & |b-c| \leqq a \leqq b+c, \\
\frac{1}{a}, & a \geqq b+c .\end{cases}
\end{aligned}
$$

On replacing $b$ by $\left(\eta^{2}-z^{2}\right)^{\frac{1}{2}}$ and $c$ by $r$ and considering the three ranges appearing in (2.8) in relation to the limits of integration with respect to $\eta$ in (2.7), it is found that the "slab" $0 \leqq z \leqq c t$ divides up into a number of regions in which the velocity potential $\phi$ takes different forms. There are six regions in all and their positions at various times are shown in Fig. 1. Four regions are present when $0<t<a / c$, five when $a / c<t<2 a / c$ and all six when $t>2 a / c$.

Let

$$
\rho_{1}=\left\{z^{2}+|r-a|^{2}\right\}^{\frac{1}{2}}, \rho_{2}=\left\{z^{2}+(r+a)^{2}\right\}^{\frac{1}{2}}
$$

be respectively the least and greatest distances of a typical point of the fluid from the edge of the piston and let

$$
k(r, z ; \eta)=\frac{1}{\pi} \cos ^{-1}\left\{\frac{\eta^{2}-z^{2}+r^{2}-a^{2}}{2 r\left(\eta^{2}-z^{2}\right)^{\frac{1}{2}}}\right\} .
$$

Then the boundaries of the regions and the expressions for $\phi$ obtained by combining equations (2.7) and (2.8) are as follows.

Region $1 \quad 0 \leqq r \leqq a, z \leqq c t \leqq \rho_{1}$,

Region $2 \quad 0 \leqq r \leqq a, \rho_{1} \leqq c t \leqq \rho_{2}$,

$$
\phi(r, z, t)=-\int_{z}^{c t} v(t-\eta / c) d \eta
$$

$$
\phi(r, z, t)=-\int_{z}^{\rho_{1}} v(t-\eta / c) d \eta-\int_{\rho_{1}}^{c t} v(t-\eta / c) k(r, z ; \eta) d \eta .
$$

Region $3 \quad 0 \leqq r \leqq a, c t \geqq \rho_{2}$,

$$
\phi(r, z, t)=-\int_{z}^{\rho_{1}} v(t-\eta / c) d \eta-\int_{\rho_{1}}^{\rho_{2}} v(t-\eta / c) k(r, z ; \eta) d \eta .
$$

Region $4 \quad r \geqq a, 0 \leqq c t \leqq \rho_{1}$,

$$
\phi(r, z, t)=0 .
$$

Region $5 \quad r \geqq a, \rho_{1} \leqq c t \leqq \rho_{2}$,

$$
\phi(r, z, t)=-\int_{\rho_{1}}^{c t} v(t-\eta / c) k(r, z ; \eta) d \eta .
$$



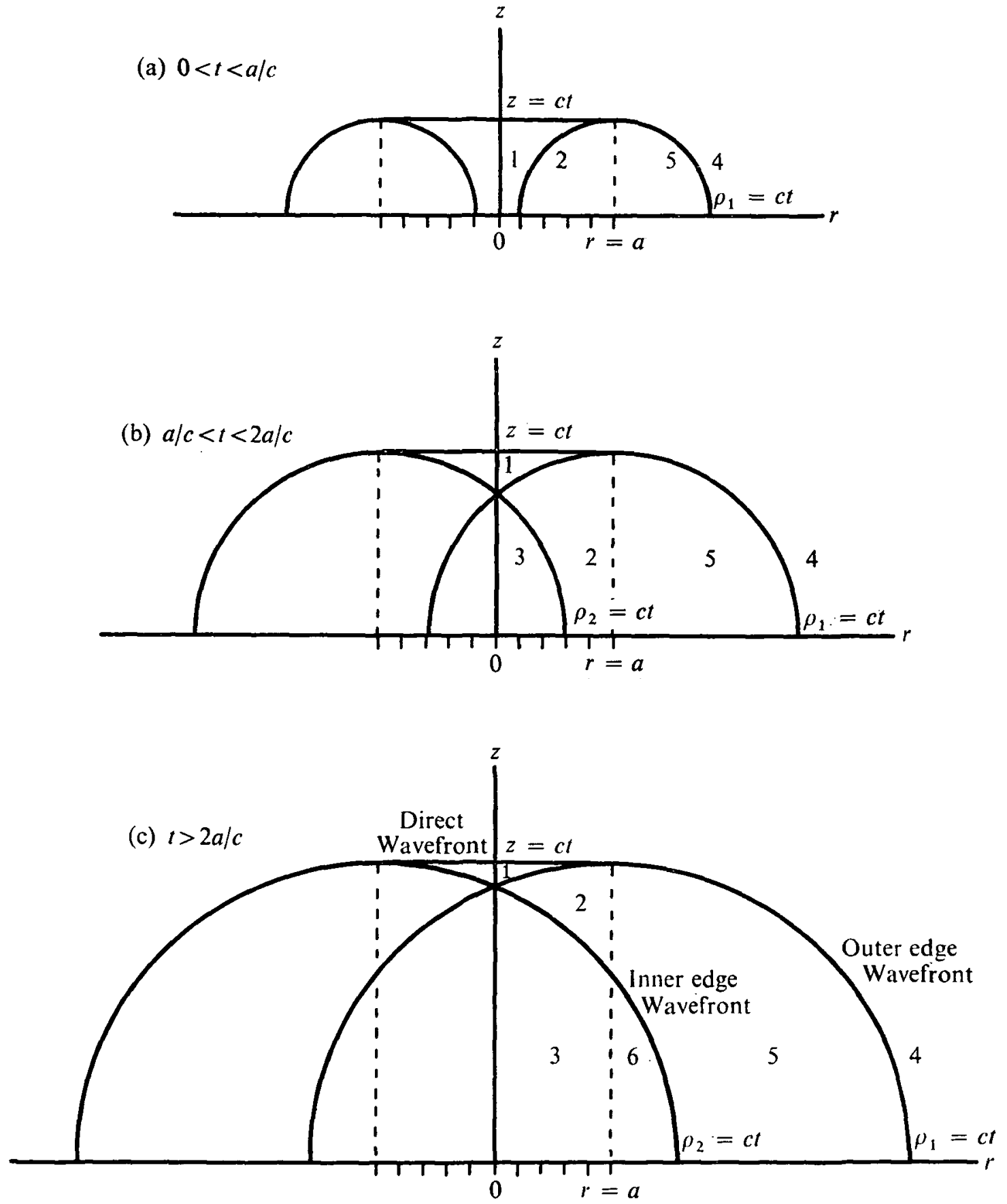

Fig. 1. Structure of the pulse: (a) $0<t<a / c$; (b) $a / c<t<2 a / c$; (c) $t>2 a / c$. 
Region $6 \quad r \geqq a, c t \geqq \rho_{2}$,

$$
\phi(r, z, t)=-\int_{\rho_{1}}^{\rho_{2}} v(t-\eta / c) k(r, z ; \eta) d \eta .
$$

The method used above of reducing the integral (2.6) to expressions involving only definite integrals has been employed by Mitra (5) and Eason (6) in the solution of elastic wave problems which have close mathematical similarities with the problem under consideration.

It remains to verify that the tentative solution (2.11) in fact satisfies the initial-boundary-value problem (2.1) to (2.3), and for this purpose we suppose that the excitation function $v(t)$ is twice continuously differentiable on an interval $0 \leqq t<T$, where $T$ is sufficiently large.

First, recalling equations (2.9) and (2.10), we observe that the partial derivatives $k_{r}(r, z ; \eta)$ and $k_{z}(r, z ; \eta)$ of $k$ with respect to $r$ and $z$ have integrable singularities at $\eta=\rho_{1}$ and $\eta=\rho_{2}$. The determination of the first partial derivatives $\partial \phi / \partial r, \partial \phi / \partial z$ and $\partial \phi / \partial t$ by direct differentiation is therefore permissible in each region and it is a simple matter to verify that the subsidiary conditions (2.2), (2.3) are satisfied.

Inspection of equations (2.11) shows that $\phi$ is a continuous function of $r, z$ and $t$ on the domain

$$
\mathscr{H}=\{(r, z, t): r \geqq 0, z \geqq 0, t \geqq 0\}
$$

and it is found that $\partial \phi / \partial r$ also has this property. The partial derivatives $\partial \phi / \partial z$ and $\partial \phi / \partial t$ are continuous on

$$
\mathscr{S}=\{(r, z, t): r \geqq 0,0 \leqq z \leqq c t, t \geqq 0\}
$$

and their domain of continuity extends to $\mathscr{H}$ if (and only if) $v(0)=0$. If this condition is violated, the expressions (2.11) do not constitute a solution of the problem (2.1) to (2.3) in the strict sense, but may be regarded as solving this problem in some generalised sense. We go on to show in $\S 3$ that the properties of $v(t)$ at $t=0$ determine the nature of the discontinuities occurring on the wavefronts $z=c t(r \leqq a)$ and $\rho_{1}=c t, \rho_{2}=c t$.

The second partial derivatives $k_{r r}(r, z ; \eta)$ and $k_{z z}(r, z ; \eta)$ of $k$ have nonintegrable singularities at $\eta=\rho_{1}$ and $\eta=\rho_{2}$ and the calculation of $\partial^{2} \phi / \partial r^{2}$, $\partial^{2} \phi / \partial z^{2}$ and $\partial^{2} \phi / \partial t^{2}$ by direct differentiation therefore leads to divergent integrals. A procedure which avoids this difficulty is to make the substitutions

$$
\begin{aligned}
& k_{r}(r, z ; \eta)=-\frac{\left(\eta^{2}-z^{2}\right)\left(\eta^{2}-z^{2}-r^{2}-a^{2}\right)}{\eta r\left(\eta^{2}-z^{2}-r^{2}+a^{2}\right)} k_{\eta}(r, z ; \eta), \\
& k_{z}(r, z ; \eta)=-\frac{z}{\eta} k_{\eta}(r, z ; \eta),
\end{aligned}
$$

in the expressions for $\partial \phi / \partial r$ and $\partial \phi / \partial z$ and then use integration by parts to replace the factor $k_{\eta}$ by $k$ in the integrands. Direct differentiation is then valid and the verification that $\phi$ satisfies the wave equation $(2.1)$ in each region is completed by a straightforward, but tedious, calculation. 
The second partial derivatives of $\phi$ are continuous on $\mathscr{P}$ if and only if $v(0)=0:$ if, in addition, $v^{\prime}(0)=0$ they are continuous on $\mathscr{H}$.

\section{The structure of the pulse}

We have shown in $\S 2$ that at time $t$ the region of disturbed fluid is the union of Regions 1, 2, 3, 5 and 6. The outer boundary of this composite region and the internal boundaries separating Regions 1 and 2, 2 and 3, and 5 and 6 are wavefronts and it is instructive to confirm the structure of the pulse, as given by the analysis of $\$ 2$ and illustrated in Fig. 1, by the methods of geometrical acoustics (11).

The pulse is made up of two components which we term the direct wave and the edge wave (cf. 7). The direct wave arises from the motion of the piston and is confined to the cylindrical region $0 \leqq r \leqq a$, being bounded in front by the plane direct wavefront $z=c t(0 \leqq r \leqq a)$ and at the rear by the piston. The edge wave is due to the motion of the edge of the piston relative to the baffle and its outer boundary is the set of all points distant $c t$ from the edge. This edge wavefront is a half-torus having the edge of the piston as its centre circle and connecting to the perimeter of the direct wavefront as shown in Fig. 1. When $t=a / c$ the edge wave begins to overlap itself and at subsequent times it is necessary to distinguish between outer and inner edge wavefronts.

It is of interest to note that the solution of the problem (2.1) to (2.3) in the limiting case $a \rightarrow \infty$, namely

$$
\phi(z, t)=-\int_{z}^{c t} v(t-\eta / c) d \eta H(t-z / c),
$$

coincides in Region 1 with the solution found in $\$ 2$ (see equation (2.11a)). This is because the fluid in Region 1 is unaware of the size of the piston having not yet been reached by a signal from its edge (the edge wave).

In the remainder of this section, we investigate the nature of the direct and edge wavefronts by examining the discontinuities in the partial derivatives of $\phi$ which occur on them. In view of the compatibility conditions connecting discontinuities (jumps) in partial derivatives on a singular surface, it is only necessary to consider the behaviour of one partial derivative of given order. The most convenient choice is the time derivative.

The direct wavefront divides Region 1 from the undisturbed fluid ahead of the pulse. In Region 1 we have, from equation (2.11a), assuming that $v(t)$ is sufficiently often differentiable,

$$
\frac{\partial^{n} \phi}{\partial t^{n}}=-c v^{(n-1)}(t-z / c), \quad n=1,2, \ldots
$$

With the usual notation for jumps, the discontinuity in $\partial^{n} \phi / \partial t^{n}$ on the direct wavefront is hence

$$
\left[\frac{\hat{o}^{n} \phi}{\partial t^{n}}\right]=c v^{(n-1)}(0), \quad n=1,2, \ldots
$$


It follows that if the derivative of the excitation function $v(t)$ of lowest order which does not vanish at $t=0$ is $v^{(N)}(t)(N \geqq 0)$, then the direct wavefront is a singular surface of order $N+1$. In particular, if $N=0$ (i.e. $v(0) \neq 0$ ) the direct wavefront is a shock wave, and if $N=1$ (i.e. $v(0)=0, v^{\prime}(0) \neq 0$ ) it is an acceleration wave.

Turning to the edge wavefront, we require expressions for $\partial^{n} \phi / \partial t^{n}$ in Regions 2 to 6 . These are derived from equations (2.11b to $\mathrm{f}$ ) as follows.

\section{Region 2}

$$
\begin{array}{r}
\frac{\partial^{n} \phi}{\partial t^{n}}=c v^{(n-1)}\left(t-\rho_{1} / c\right)-c v^{(n-1)}(t-z / c)-\sum_{s=1}^{n} c^{s} v^{(n-s)}(0)\left\{\frac{\partial^{s-1}}{\partial \eta^{s-1}} k(r, z ; \eta)\right\}_{\eta=c t} \\
-\int_{\rho_{1}}^{c t} v^{(n)}(t-\eta / c) k(r, z ; \eta) d \eta .
\end{array}
$$

Region 3

$\frac{\partial^{n} \phi}{\partial t^{n}}=c v^{(n-1)}\left(t-\rho_{1} / c\right)-c v^{(n-1)}(t-z / c)-\int_{\rho_{1}}^{\rho_{2}} v^{(n)}(t-\eta / c) k(r, z ; \eta) d \eta$.

\section{Region 4}

$\frac{\partial^{n} \phi}{\partial t^{n}}=0$.

\section{Region 5}

$\frac{\partial^{n} \phi}{\partial t^{n}}=-\sum_{s=1}^{n} c^{s} v^{(n-s)}(0)\left\{\frac{\partial^{s-1}}{\partial \eta^{s-1}} k(r, z ; \eta)\right\}_{\eta=c t}-\int_{\rho_{1}}^{c t} v^{(n)}(t-\eta / c) k(r, z ; \eta) d \eta$.

\section{Region 6}

$\frac{\partial^{n} \phi}{\partial t^{n}}=-\int_{\rho_{1}}^{\rho_{2}} v^{(n)}(t-\eta / c) k(r, z ; \eta) d \eta$,

where in each case $n=1,2, \ldots$ Using the results

$$
k\left(r, z ; \rho_{1}\right)=\left\{\begin{array}{l}
1,0 \leqq r<a, \\
0, r>a,
\end{array} \quad k\left(r, z ; \rho_{2}\right)=0,\right.
$$

which follow from equations (2.9) and (2.10), together with (3.1), we obtain for the outer edge wavefront, $\rho_{1}=c t$,

$$
\left[\frac{\partial^{n} \phi}{\partial t^{n}}\right]=\sum_{s=2}^{n} c^{s} v^{(n-s)}(0) \lim _{\eta \rightarrow \rho_{1}} \frac{\partial^{s-1}}{\partial \eta^{s-1}} k(r, z ; \eta),
$$

and for the inner edge wavefront, $\rho_{2}=c t$,

$$
\left[\frac{\partial^{n} \phi}{\partial t^{n}}\right]=-\sum_{s=2}^{n} c^{s} v^{(n-s)}(0) \lim _{\eta \rightarrow \rho_{2}} \frac{\partial^{s-1}}{\partial \eta^{s-1}} k(r, z ; \eta)
$$

where $n=1,2, \ldots$. Now all the partial derivatives of $k$ with respect to $\eta$ are 
singular at $\eta=\rho_{1}$ and $\eta=\rho_{2}$. We therefore conclude from equations (3.2) and (3.3) that if the derivative of $v(t)$ of lowest order which does not vanish at $t=0$ is $v^{(N)}(t)$, then both the inner and outer edge wavefronts are singular surfaces of order $N+2$ on which the partial derivatives of $\phi$ of order $N+2$ suffer infinite jumps. In particular, if $N=0$ (i.e. $v(0) \neq 0$ ) the edge wavefronts are infinite acceleration waves.

\section{A special case}

In this section we discuss in some detail the special case of the foregoing analysis in which $v(t)=v$, a constant. Defining the function

$$
K(r, z ; \eta)=\int_{\rho_{1}}^{\eta} k(r, z ; \zeta) d \zeta,
$$

where $k$ is given by equation (2.10), the solution of this particular case, obtained by specialising equations (2.11), can be stated in the concise form

$$
\begin{aligned}
\phi(r, z, t)= & v\left\langle\left\{z-c t+\left(c t-\rho_{1}\right) H\left(t-\rho_{1} / c\right)\right\} H(a-r)\right. \\
& -K(r, z ; c t) H\left(t-\rho_{1} / c\right) \\
& \left.-\left\{K\left(r, z ; \rho_{2}\right)-K(r, z ; c t)\right\} H\left(t-\rho_{2} / c\right)\right\rangle H(t-z / c) .
\end{aligned}
$$

It should be noted that a motion of the piston with constant velocity can be maintained for only a finite time without violating the assumptions upon which the acoustic linearisation is based. Accordingly the solution (4.1) has a limited range of validity at a given point of the fluid. If, at some time, the piston is brought to rest, a further system of wavefronts is generated having the same structure as those shown in Fig. 1. The analysis may readily be extended to include these events, but in order to preserve a maximum of simplicity we suppose here that $v$ is sufficiently small to ensure the validity of all our results.

For the particular excitation function chosen, the pressure

$$
p=p_{0}-\rho_{0} \frac{\partial \phi}{\partial t}
$$

can be evaluated in the simple closed form

$$
\begin{aligned}
p=p_{0}+\rho_{0} c v\left\langle\left\{1-H\left(t-\rho_{1} / c\right)\right\}\right. & H(a-r) \\
+ & \left.k(r, z ; c t)\left\{H\left(t-\rho_{1} / c\right)-H\left(t-\rho_{2} / c\right)\right\}\right\rangle H(t-z / c) .
\end{aligned}
$$

The nature of the pressure-time profile at a point $P(r, z)$ of the fluid depends upon the number of wavefronts which pass through that point. Two cases arise.

If $0 \leqq r \leqq a$, there are three wave arrivals at $P$. The direct wavefront arrives first, at $t=z / c$, and produces a discontinuous increase in pressure of amount $\rho_{0} c v . P$ then enters Region 1 and the pressure remains constant until 


$$
9
$$


the arrival, at $t=\rho_{1} / c$, of the outer edge wavefront. At this instant, the derivative $\partial p / \partial t$ suffers an infinite negative discontinuity. $P$ next passes into Region 2 and the pressure decreases, regaining its initial value $p_{o}$ at the instant, $t=\rho_{2} / c$, when the inner edge wavefront arrives. Thereafter $P$ is situated in Region 3 and $p=p_{0}$.

If $r>a$, there are only two wave arrivals at $P$. Prior to the arrival, at $t=\rho_{1} / c$, of the outer edge wavefront, $P$ is in Region 4 and $p=p_{0}$. The first arrival is associated with an infinite positive jump in $\partial p / \partial t$ and the arrival, at $t=\rho_{2} / c$, of the inner edge wavefront also results in an infinite positive jump in this derivative. During the intervening period, $P$ lies in Region 5 and the pressure increases to a maximum value of $p_{0}+\left(\rho_{0} c v / \pi\right) \sin ^{-1}(a / r)$, reached at time $\left(r^{2}-a^{2}+z^{2}\right)^{\frac{1}{2}} / c$, before decreasing to the initial value $p_{0}$. For $t>\rho_{2} / c, P$ is situated in Region 6 and $p=p_{0}$.

Curves showing the variation of the reduced pressure increment $\left(p-p_{0}\right) / \rho_{0} c v$ with the reduced time $c t / a$ at the points $r=\frac{1}{2} a, z=a$ and $r=\frac{3}{2} a, z=a$ are presented in Fig. 2.

Also of interest is the distribution of pressure on the boundary $z=0$, and curves showing the variation of $\left(p-p_{0}\right) / \rho_{0} c v$ with $r / a$ at various reduced times are given in Fig. 3. For $0 \leqq t<a / c$, part of the piston adjoins Region 1 and is subject to a uniform pressure increment of amount $\rho_{0} c v$. In the annular region $a-c t \leqq r \leqq a+c t$, the pressure decreases as $r$ increases, reaching its initial value $p_{0}$ on the outer edge wavefront. For $t>a / c$, there is a circular region of uniform pressure $p_{0}$ associated with Region 3 and later with Regions 3 and 6 . In the annulus $c t-a \leqq r \leqq c t+a$, the pressure increases, with increasing $r$, to a maximum value of $p_{0}+\left(\rho_{0} c v / \pi\right) \sin ^{-1}(a / c t)$ at $r=\left(c^{2} t^{2}-a^{2}\right)^{\frac{1}{2}}$ before decreasing to its initial value $p_{0}$ on the outer edge wavefront. The change-over between the two types of pressure distribution occurs at $t=a / c$ when the edge wave begins to overlap and is associated with a sudden drop in pressure of amount $\rho_{o} c v$ at $O$. Curves indicating the manner in which the transition takes place are shown in Fig. 3c.

It is evident from Figs. 2 and 3 that the sound pulse produced by pushing the piston into the fluid with constant speed is purely compressive in the sense that $p \geqq p_{0}$ for all $t \geqq 0$ at all points of the flow. This property is apparently due to the presence of a plane baffle. In the problem studied in (7) the piston and the baffle are convex towards the fluid, and it is found that the sound pulse produced by an outward motion of the piston with constant speed is not entirely compressive on account of the appearance in the flow of diffracted waves $((7), \S 8)$. If the baffle is removed there results a situation having some affinity with a problem discussed by D. S. Jones ((12), pp. 520-522). $\dagger$ Jones considers the head-on impact of a plane sound pulse with the end-face of a rigid semi-infinite circular cylinder of radius $a$ and obtains an approximate expression for the variation with time of the mean pressure increment, say $\vec{q}$,

$\uparrow$ The authors are indebted to a referee for this reference. 
on the end-face. Taking the incident sound pulse to be a uniform shock wave producing a pressure increment $q$ at all points behind its front, $\bar{q}=2 q$ at the instant of impact ( $t=0)$ and $\bar{q} \rightarrow q$ as $t \rightarrow \infty$. $\bar{q}$ decreases rapidly to a minimum value, less than $q$, at $t=2 a / c$ and thereafter approaches the steady-state value $q$ from below. The scattered pulse is, therefore, not wholly compressive.

One of us, G. E. Tupholme, wishes to thank the Science Research Council for the award of a Research Studentship.

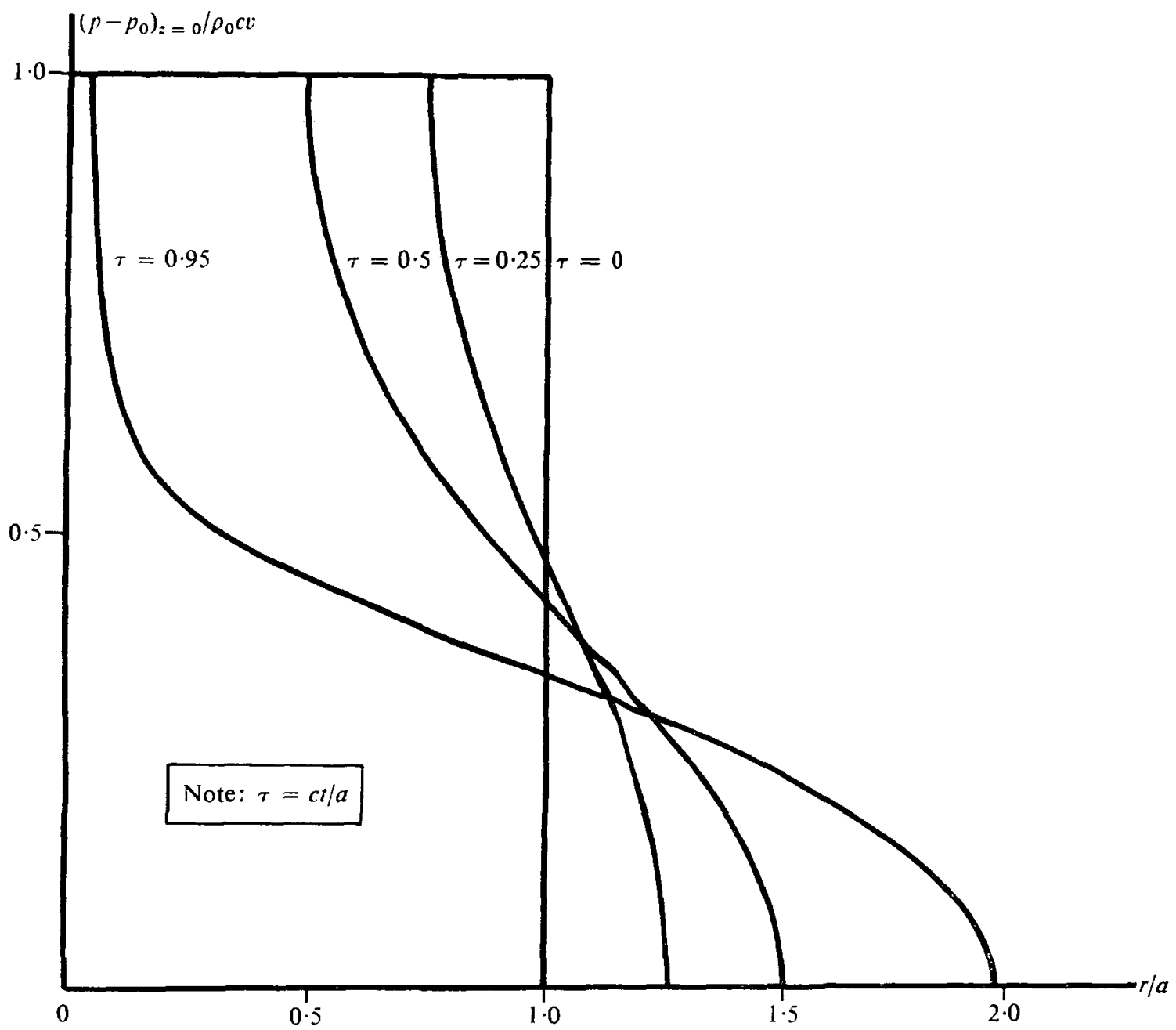

FIG. 3A.-Pressure distribution on $z=0$ : (a) $0<t<a / c$; (b) $t>a / c$; (c) $t=0.99 a / c$ and 


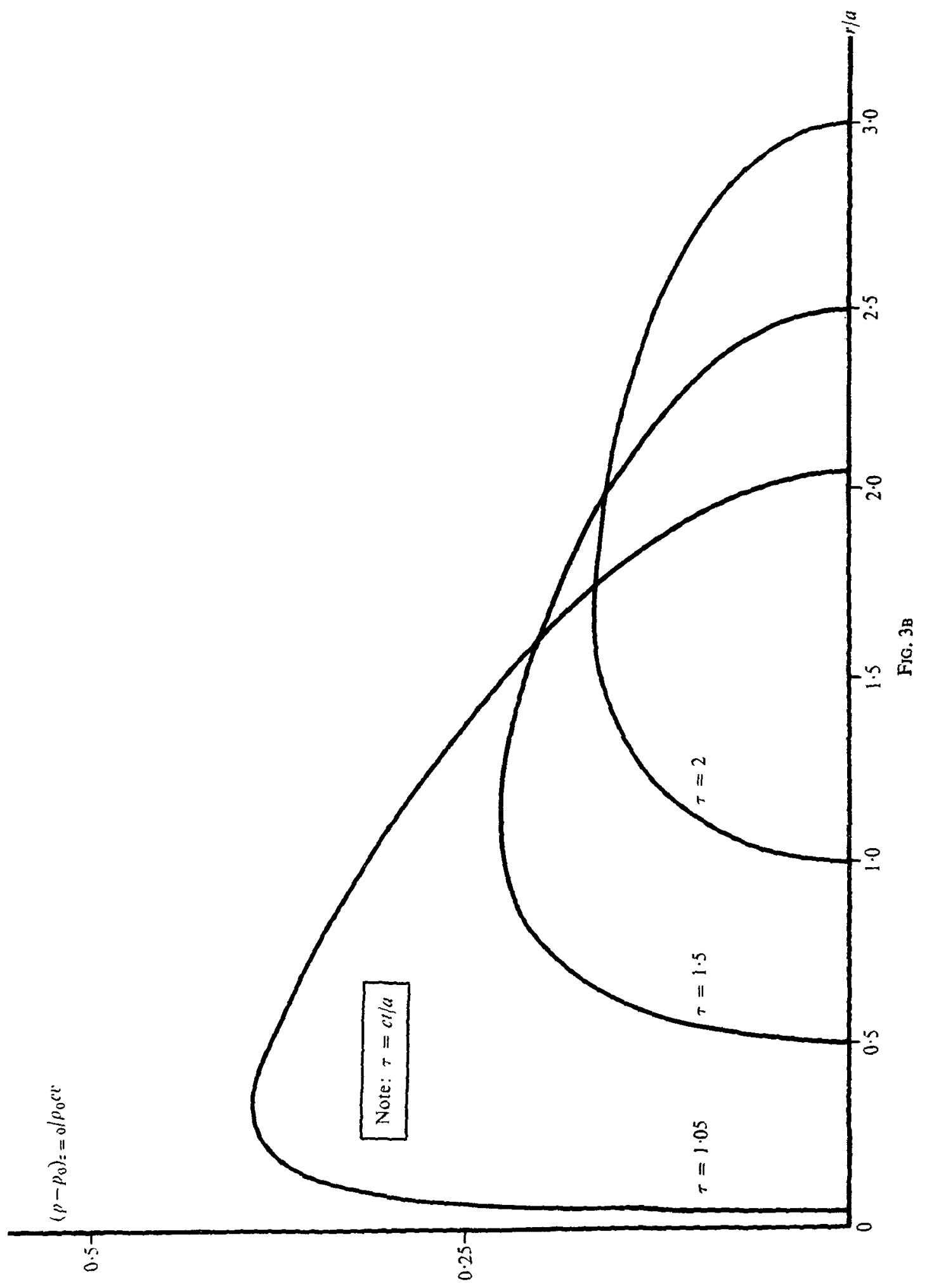




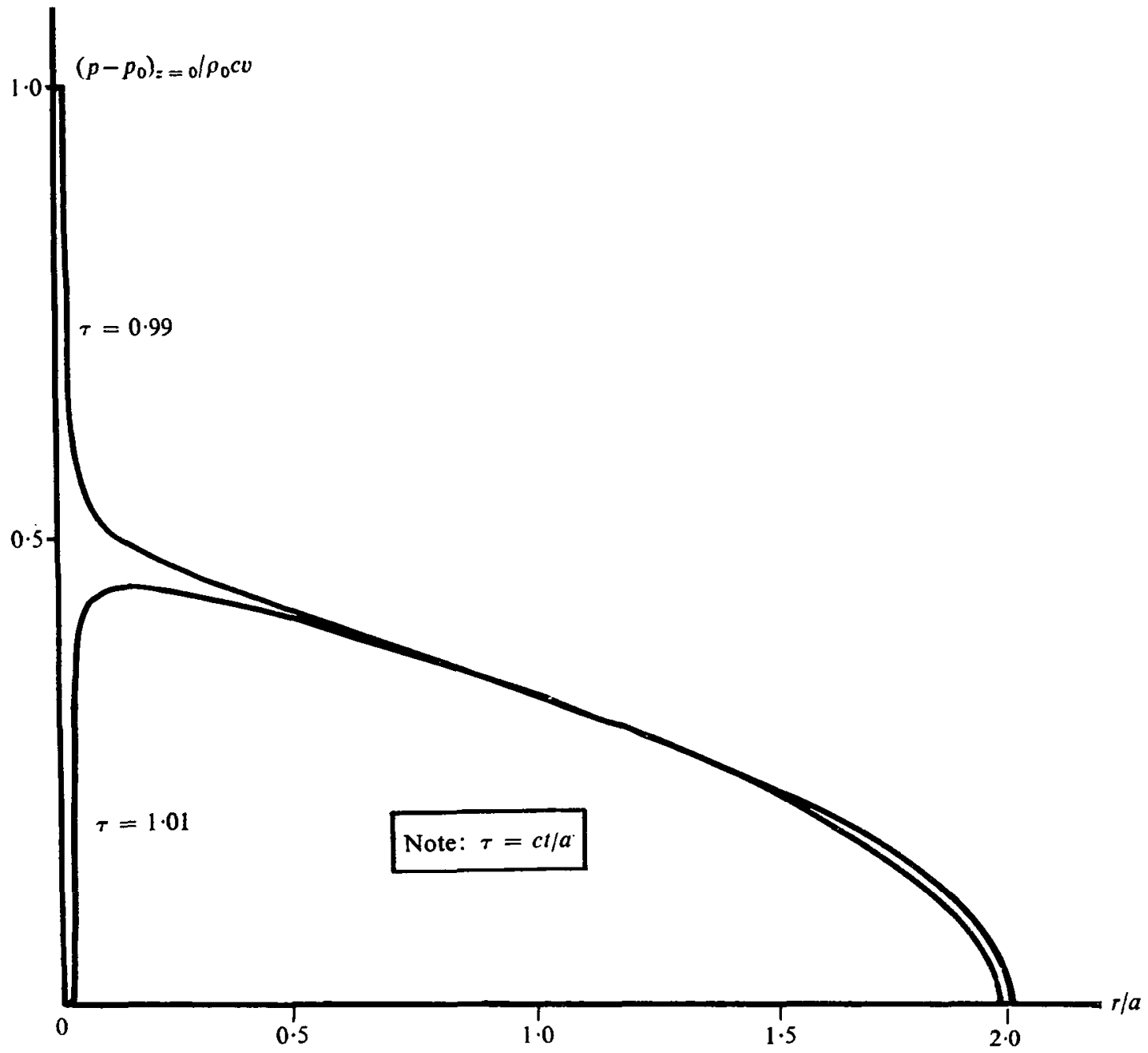

FIG. 3C

\section{REFERENCES}

(1) C. P. Wells and A. Leitner, Quart. Appl. Math. 15 (1957), 430-434.

(2) F. Oberhettinger, J. Res. Nat. Bur. Standards, 65B (1961), 1-6.

(3) F. Oberhettinger, J. Res. Nat. Bur. Standards, 65B (1961), 203-204.

(4) A. O. Williams, JR., J. Acoust. Soc. Amer. 36 (1964), 2408-2410.

(5) A. K. Mirra, Proc. Nat. Inst. Sci. India, 27 A (1961), 470-475.

(6) G. EASON, Quart. J. Mech. Appl. Math. 17 (1964), 279-292. 
(7) G. E. Tupholme, Proc. Cambridge Philos. Soc, to be published.

(8) I. N. SNEddon, Fourier Transforms (McGraw-Hill, 1951).

(9) A. ERDÉlyi et al. (editors), Tables of Integral Transforms, Vol. 1 (McGraw-Hill, 1954).

(10) G. N. WATSON, A Treatise on the Theory of Bessel Functions, 2nd edn. (Cambridge University Press, 1944).

(11) J. B. Keller, Proc. 8th Symp. Appl. Math. Amer. Math. Soc. (McGraw-Hill, 1958), pp. 27-52.

(12) D. S. Jones, Philos. Trans. Roy. Soc. London Ser. A. 247 (1955), 499-528.

School of Mathematics and Physics, University of East Anglia, Norwich 